nephron

Practice
Nephron 2019;143:197-201

DOI: $10.1159 / 000502456$
Received: April 29, 2019

Accepted after revision: August 1, 2019

Published online: August 21, 2019

\title{
Renal Injury During Preclampsia: Role of Extracellular Vesicles
}

\author{
Fabiola Pagani Vincenzo Cantaluppi \\ Nephrology and Kidney Transplantation Unit, Department of Translational Medicine and Center for Autoimmune and \\ Allergic Diseases (CAAD), University of Piemonte Orientale (UPO), "Maggiore della Carità" University Hospital, Novara, Italy
}

\author{
Keywords \\ Preeclampsia - Acute kidney injury - Extracellular vesicles . \\ Endothelial dysfunction
}

\begin{abstract}
Preeclampsia (PE) represents an important cause of acute kidney injury during pregnancy with a high rate of both maternal and fetal morbidity and mortality. The presence of endothelial dysfunction is the main cause of PE development; however, the pathogenic mechanisms are not still fully elucidated. A plethora of harmful mediators are released in response to endothelial damage: these circulating molecules (soluble fms-like tyrosine kinase 1, endothelin-1) can exert antiangiogenic and vasoconstrictive effects on distant organs including the kidney. In this scenario, extracellular vesicles (EVs), microparticles released by different types of activated cells and involved in intercellular communication, are produced both in normal pregnancy and PE. The aim of this review is to summarize the modern concepts of PE-associated kidney damage and the potential role of EV in these detrimental mechanisms.

(c) 2019 S. Karger AG, Basel
\end{abstract}

\section{Introduction}

Preeclampsia (PE) is a hypertensive disorder complicating up to $8 \%$ of pregnancies worldwide and represents a relevant cause of both maternal and fetal morbidity and mortality. Moreover, PE represents one of the possible causes of acute kidney injury in the 2nd and 3rd trimester of pregnancy [1] (Fig. 1). Acute kidney injury in PE is relatively uncommon (1\%) but represent a criterion of illness severity [1]. The aim of this review is to summarize the more recent concepts of PE-associated kidney damage and the potential role of extracellular vesicles (EV) in these pathogenic mechanisms.

\section{Endothelial Dysfunction and Kidney Injury}

The pathogenic mechanisms of PE-associated kidney injury have not been fully elucidated; however, the most common hypothesis is that abnormal placentation and endothelial dysfunction both play an essential role. A defect in remodeling of placental spiral arteries during trophoblast invasion through the uterine wall causes an altered perfusion of intervillous space. Consequently, placental ischemic damage leads to the release of antiangiogenic and vasoconstrictive factors into the maternal circulation [2]: Soluble fms-like tyrosine kinase 1

\section{KARGER}

(c) 2019 S. Karger AG, Basel
Contribution from the Acute Kidney Injury (AKI) and CRRT 2019 Symposium at the 24th International Conference on Advances in Critical Care Nephrology, Manchester Grand Hyatt, San Diego, CA, USA, February 26 - March 1, 2019. This symposium was supported in part by the NIDDK-funded University of Alabama at BirminghamUniversity of California San Diego O'Brien Center for AKI Research (P30DK079337). 


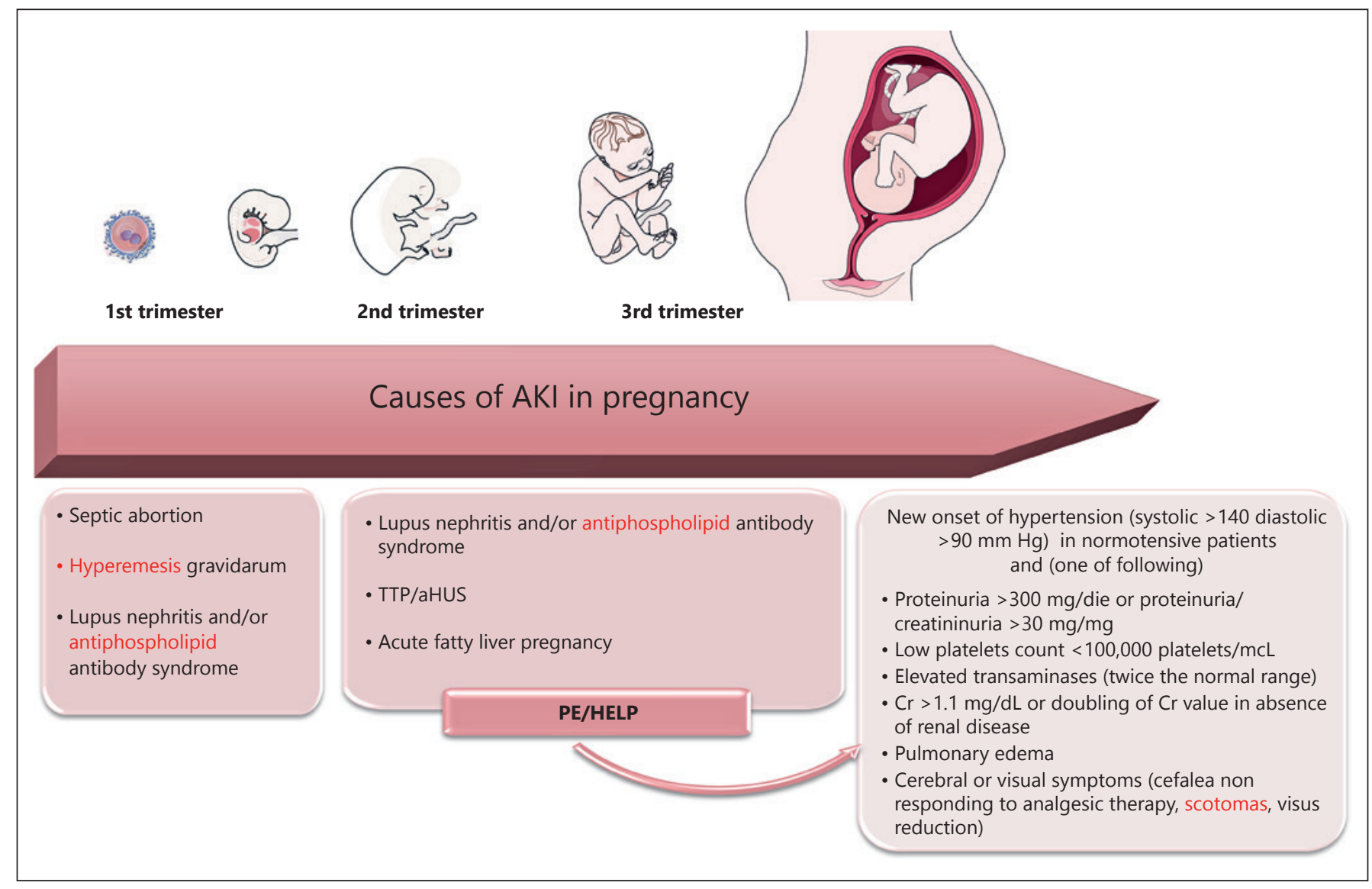

Fig. 1. Causes of AKI during pregnancy. PE is a possible cause of renal injury from the 20th gestational week. AKI, acute kidney injury; TTP, thrombotic thrombocytopenic purpura; aHUS, atypical hemolytic uremic syndrome; PE, preeclampsia; HELP, heparinmediated extracorporeal low-density lipoprotein precipitation; $\mathrm{Cr}$, serum creatinine.
(sFlt-1) can bind vascular endothelial growth factor and placental growth factor inhibiting interaction with their specific receptors. When injected into pregnant mice, sFlt-1 can cause hypertension, albuminuria, and histological changes commonly found in PE [3]. In addition, soluble endoglin (sEng) is a circulating coreceptor of the proangiogenic factor TGF $\beta$ known to antagonize the binding between TGF $\beta$ and its vascular receptor [4].

In late forms of $\mathrm{PE}$ (after 34th gestational week), a preexisting endothelial dysfunction due to oxidative stress and/or metabolic alterations (hypertension, obesity, diabetes, etc.) is more frequent. The inadequate adaptive capacity in response to the physiological stress induced by pregnancy is responsible for the development of clinical evident microvascular damage. However, multiple causes of endothelial dysfunction can coexist [2, 5], including activation of the complement cascade [6] and lack of vascular regeneration due an inadequate re- lease of functional endothelial progenitor cells from the bone marrow [7].

The mechanisms of glomerular permeability and proteinuria are also regulated by the accurate interplay between endothelial cells and podocytes. Podocyturia is a potential earlier marker of glomerular damage, and recent papers showed a correlation between the number of podocytes lost in urine and proteinuria [8]. In addition, a reduced expression of the podocyte slit diaphragm protein nephrin has been described: it has been postulated that circulating factors during PE may induce an increased release of endothelin-1 (ET1) from glomerular endothelial cells. ET1 is a strong vasoconstrictor agent that leads to consequent nephrin shedding from podocytes [9]. Moreover, vascular endothelial growth factor inhibition correlates with ET1 increased production by endothelial cells [9], suggesting a pathogenic role of sFlt-1 in the development of proteinuria. ET1 may also repre- 


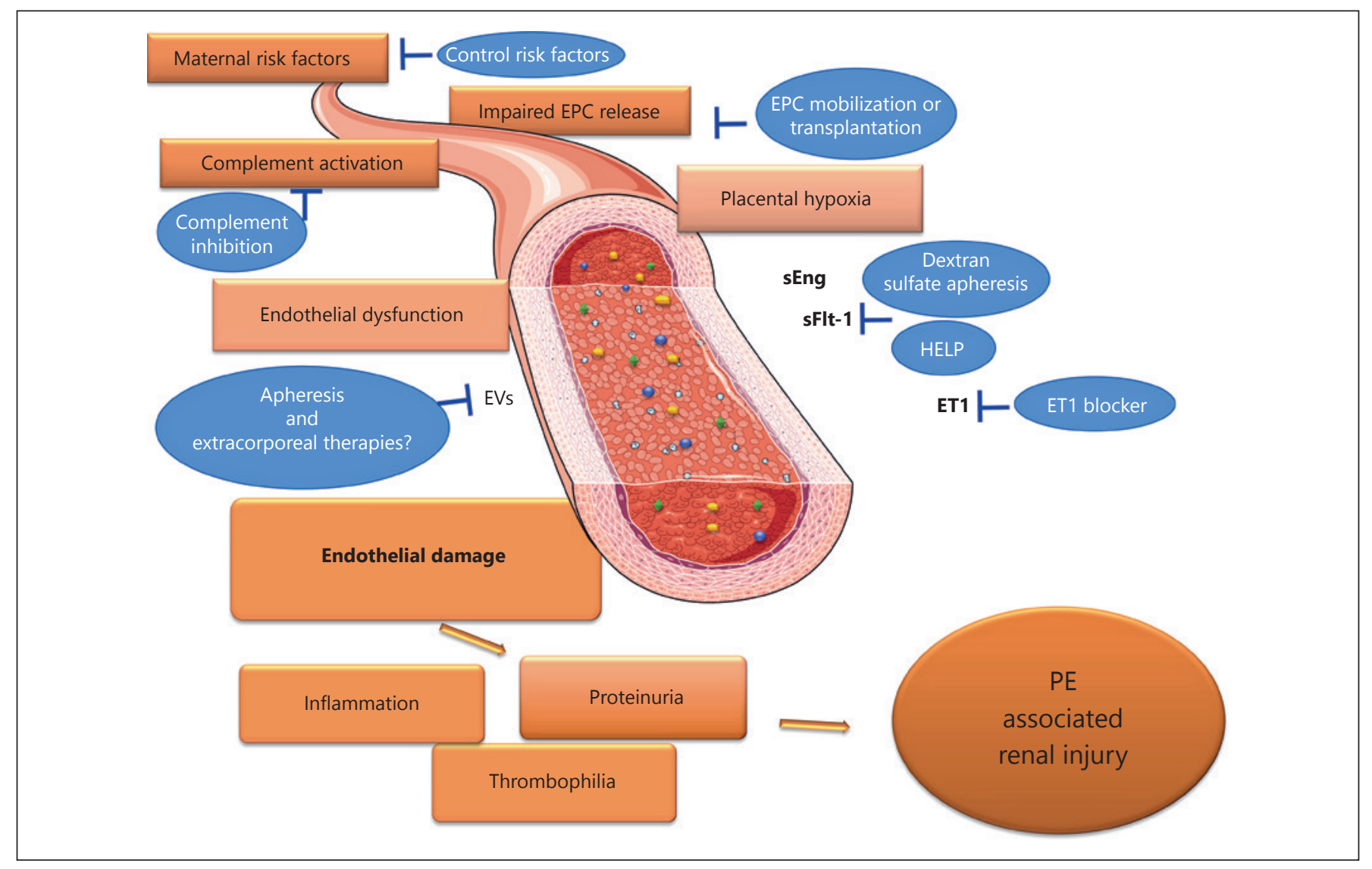

Fig. 2. Main causes of PE-associated renal injury and potential therapeutic options. Endothelial dysfunction plays a key role in PE-associated renal injury. In the blue circle boxes are illustrated possible future therapeutic approaches. EPC, endothelial progeni-

sent a possible link between endothelial dysfunction and podocyte damage; indeed, in vitro studies suggest that endogenous ET-1 can lead to glomerulosclerosis and proteinuria and that podocyte apoptosis and glomerular damage could be reversed by using ET1 blockers [10].

\section{Role of EVs}

EVs represent a key mechanism of cell-to-cell communication preserved between species and they are formed by 3 main families: Exosomes $(50-150 \mathrm{~nm})$ produced in a constitutive manner and secreted by exocytosis; Microvesicles or Shedding Vesicles (100-1,000 nm) directly released from activated cells by a membrane sorting mechanism in response to the increase of intracellular calcium; Apoptotic bodies that are cellular residues derived from apoptosis. EVs communicate with target cells tor cells; EVs, extracellular vesicles; sEng, soluble endoglin; sFlt-1, soluble fms-like tyrosine kinase 1; HELP, heparin-mediated extracorporeal low-density lipoprotein precipitation; ET1, endothelin-1; PE, preeclampsia. via receptor-ligand interaction, by fusion with cell membrane and cytoplasmic release of transported proteins or by direct transfer of mRNA and in particular microRNA, small noncoding RNAs able to modulate protein translation [11]. EVs of placental origin are released during both physiologic and pathologic pregnancy mainly by syncytiotrophoblasts and have a specific surface marker, the placental alkaline phosphatase. Cellular targets of placental derived-EV are different but mostly related with maternal immune system aimed at inducing and maintaining a state of immunotolerance with the fetus (by an increased anti-inflammatory cytokine production, a decreased proinflammatory cytokine release, a lower class II MHC protein expression on macrophages, a downregulation of T cell response) [12, 13]. Furthermore, during $\mathrm{PE}, \mathrm{EV}$ concentration is increased compared to physiological pregnancies, but there is not agreement in literature on the presence of a direct correlation between 
EV concentration and severity of disease $[12,13]$. EVs released from endothelial cells and activated leukocytes or platelets can also be identified within maternal circulation. It has also been shown that syncytiotrophoblast-derived EV s have a higher tissue factor activity, can promote platelet aggregation, and have an antiangiogenic and hypertensive effect on endothelial cells [13]. Of interest, it has been shown that exosomes isolated from preeclamptic patients express sFlt- 1 and sEng and that they can impair angiogenesis reducing proliferation and migration of endothelial cells in vitro [14]. Furthermore, urine EVs may have a potential role as PE biomarkers: Gilani et al. [15] recently demonstrated that in comparison to healthy pregnancy, urine from women with PE contained a high ratio of podocin-positive to nephrin-positive EVs, suggesting that urine EVs may reflect $\mathrm{PE}$-associated alteration of podocyte protein expression.

\section{Possible Future Therapeutic Approaches}

PE therapy is basically related to the control of hypertension, proteinuria, and renal function trying to minimize maternal and fetal risks. Recently, Thadhani et al. [16] demonstrated the efficient removal of sFlt-1 by dextran sulfate apheresis in 11 very preterm women (23-32th gestational week) resulting in a reduction of proteinuria and an apparent pregnancy extension without major maternal adverse events or fetal consequences. In addition, a deranged lipoprotein metabolism is thought to impact endothelial and placental function in the course of PE; in this setting, lipoprotein-apheresis by heparin-mediated extracorporeal low-density lipoprotein precipitation has been shown to prolong gestation even without significant alteration of sFlt-1 levels and sFlt-1/placental growth factor ratio [17].

The main causes of PE-associated kidney injury and possible therapeutic approaches are briefly summarized in Figure 2. Apart from the abovementioned apheresis techniques, in the near future new drugs aimed to inhibit the activation of the complement cascade and to enhance endothelial progenitor cell release from the bone marrow may also prevent or limit PE-associated endothelial injury and renal dysfunction.

\section{Conclusion}

The development of proteinuria and kidney injury during $\mathrm{PE}$ is correlated with the presence of endothelial dysfunction. Antiangiogenetic factors such as sFlt- 1 and sEng have a well-known role in PE-associated kidney alterations: in this scenario, EVs released from placenta, microvascular endothelium, and circulating leucocytes and platelets are fascinating biomarkers of disease severity, but they may also contribute to the pathogenic mechanisms of glomerular damage. Further studies are needed to define the role of EVs in the modulation of endothelialpodocyte cross-talk within the glomeruli, a biological event that may lead to the deterioration of the filtration barrier and consequently to proteinuria. On this basis, EVs may become a potential target of future therapy in PE.

\section{Disclosure Statement}

The authors have no conflicts of interest to declare.

\section{Author Contributions}

Fabiola Pagani, MD: organization of the paper and substantial contribution to the acquisition of data. Vincenzo Cantaluppi, MD: organization of the paper and revision critically for intellectual content.

\section{References}

1 Rao S, Jim B. Acute Kidney Injury in Pregnancy: The Changing Landscape for the 21st Century. Kidney Int Rep. 2018 Feb;3(2):24757.

2 Phipps E, Prasanna D, Brima W, Jim B. Preeclampsia: Updates in Pathogenesis, Definitions, and Guidelines. Clin J Am Soc Nephrol. 2016 Jun;11(6):1102-13

3 Maynard SE, Min JY, Merchan J, Lim KH, Li J, Mondal S, et al. Excess placental soluble fms-like tyrosine kinase 1 (sFlt1) may contribute to endothelial dysfunction, hyperten- sion, and proteinuria in preeclampsia. J Clin Invest. 2003 Mar;111(5):649-58.

4 Venkatesha S, Toporsian M, Lam C, Hanai J, Mammoto T, Kim YM, et al. Soluble endoglin contributes to the pathogenesis of preeclampsia. Nat Med. 2006 Jun;12(6): 642-9.

5 Lisonkova S, Joseph KS. Incidence of preeclampsia: risk factors and outcomes associated with early- versus late-onset disease. Am J Obstet Gynecol. 2013 Dec;209(6):544.e112.
6 Regal JF, Burwick RM, Fleming SD. The Complement System and Preeclampsia. Curr Hypertens Rep. 2017 Oct;19(11):87.

7 Attar A, Monabati A, Parsanezhad ME. Endothelial progenitor cell subsets and preeclampsia: findings and controversies. J Chin Med Assoc. 2017 Oct;80(10):615-22.

8 Craici IM, Wagner SJ, Bailey KR, Fitz-Gibbon PD, Wood-Wentz CM, Turner ST, et al. Podocyturia predates proteinuria and clinical features of preeclampsia: longitudinal prospective study. Hypertension. 2013 Jun;61(6):1289-96. 
9 Collino F, Bussolati B, Gerbaudo E, Marozio L, Pelissetto S, Benedetto C, et al. Preeclamptic sera induce nephrin shedding from podocytes through endothelin-1 release by endothelial glomerular cells. Am J Physiol Renal Physiol. 2008 May;294(5):F1185-94.

10 Craici IM, Wagner SJ, Weissgerber TL, Grande JP, Garovic VD. Advances in the pathophysiology of pre-eclampsia and related podocyte injury. Kidney Int. 2014 Aug;86(2): 275-85.

11 Camussi G, Deregibus MC, Bruno S, Cantaluppi V, Biancone L. Exosomes/microvesicles as a mechanism of cell-to-cell communication. Kidney Int. 2010 Nov;78(9):838-48.

12 Adam S, Elfeky O, Kinhal V, Dutta S, Lai A, Jayabalan $\mathrm{N}$, et al. Review: fetal-maternal communication via extracellular vesicles Implications for complications of pregnancies. Placenta. 2017 Jun;54:83-8.

13 Tannetta D, Masliukaite I, Vatish M, Redman C, Sargent I. Update of syncytiotrophoblast derived extracellular vesicles in normal pregnancy and preeclampsia. J Reprod Immunol. 2017 Feb;119:98-106.

14 Chang X, Yao J, He Q, Liu M, Duan T, Wang K. Exosomes From Women With Preeclampsia Induced Vascular Dysfunction by Delivering sFlt (Soluble Fms-Like Tyrosine Kinase)-1 and sEng (Soluble Endoglin) to Endothelial Cells. Hypertension. 2018 Dec;72(6):138190.

15 Gilani SI, Anderson UD, Jayachandran $\mathrm{M}$, Weissgerber TL, Zand L, White WM, et al. Urinary Extracellular Vesicles of Podocyte Origin and Renal Injury in Preeclampsia. J Am Soc Nephrol. 2017 Nov;28(11): 3363-72.

16 Thadhani R, Hagmann H, Schaarschmidt W, Roth B, Cingoez T, Karumanchi SA, et al. Removal of Soluble Fms-Like Tyrosine Kinase-1 by Dextran Sulfate Apheresis in Preeclampsia. J Am Soc Nephrol. 2016 Mar;27(3):90313.

17 Winkler K, Contini C, König B, Krumrey B, Pütz G, Zschiedrich S, et al. Treatment of very preterm preeclampsia via heparin-mediated extracorporeal LDL-precipitation (H.E.L.P.) apheresis: the Freiburg preeclampsia H.E.L.P.-Apheresis study. Pregnancy Hypertens. 2018 Apr;12:136-43. 